\title{
Advances and prospects of genetic mapping of Verticillium wilt resistance in cotton
}

\author{
AINI Nurimanguli ${ }^{1}$, JIBRIL Abdulaziz Nuhu ${ }^{1}$, LIU Shiming ${ }^{2}$, HAN Peng ${ }^{1}$, PAN Zhenyuan ${ }^{1}$, ZHU Longfu ${ }^{1,2^{*}}$ and \\ NIE Xinhui ${ }^{*}$
}

\begin{abstract}
Verticillium wilt is one of the most important diseases affecting cotton production in China. The fungus, Verticillium dahliae, has a wide host range and a high degree of genetic variability. No resistance resources have been found in the available planting resources, thus presenting difficulties and challenges for our study. The long-term production practice shows that selection of disease-resistant varieties is the most economical and effective measure to control Verticillium wilt of cotton to reduce the yield loss and quality decline of cotton. In this paper, we summarized the genetic mapping population, the analysis method of genetic localization, the discovery, mining and cloning of disease-resistant quantitative trait loci/markers, and the analysis of their genetic functions, so as to provide information for the molecular breeding approach of disease-resistant cotton.
\end{abstract}

Keywords: Cotton Verticillium wilt, Genetic mapping, QTL

\section{Introduction}

Cotton is considered to be a major cash crop in the world and one of the largest raw materials available in agricultural industry. Cotton is also an important source of vegetable protein and edible oil. China is the world's second largest cotton producer following India, with a vast and high-yielding cotton planting area. Cotton production plays a vital role in the development of economy and society. Verticillium wilt is currently one of the most common diseases seriously effect cotton yield and quality (Song et al. 2020). It is a soil-borne fungal disease of the cotton vascular system caused primarily by Verticillium dahliae ( $V$. dahliae) infection (Fradin and Thomma 2006). Because $V$. dahliae has a broad host range and the durable nature of its soilborne microsclerotia, it can easily survive in the changing environment and host ( $\mathrm{Li}$ et al. 2017b). Verticillium wilt severity is influenced by many factors, such as the

*Correspondence: Ifzhu@mail.hzau.edu.cn; xjnxh2004130@126.com ${ }^{1}$ Key Laboratory of Oasis Ecology Agricultural of Xinjiang Bingtuan, Agricultural College, Shihezi University, Shihezi 832003, Xinjiang, China Full list of author information is available at the end of the article host resistance genes, the pathogenicity of the pathogen, and the ability of the fungus to detect resistance mechanisms. Furthermore, isolates of $V$. dahliae can be characterized as defoliating or nondefoliating based on symptoms of cotton, or as race 1 or race 2 based on the responses of different cultivars. After investigating the frequency and distribution of races and defoliation phenotypes of cotton-associated $V$. dahliae, it was find that $97.2 \%$ of isolates genotyped as defoliating were also characterized as race 2 , while $90.8 \%$ of isolates genotyped as nondefoliating were also genotyped as race 1 (Hu et al. 2015). The representative $74 \mathrm{~V}$. dahliae isolates from 28 different cotton regions in three major cotton-production areas in China were identified for their pathotypes, races, vegetative compatibility groups (VCGs) and genetic diversity by specific polymerase chain reaction. Of 74 isolates, 60 (81\%) were defoliating pathotype strains, especially in Yellow River/Yantze River Valley region. Seventy isolates belonged to race 2 , whereas no race 1 specific fragment was amplified (Zhang et al. 2019a, b). Eighty-five $V$. dahliae isolates were obtained from wilted cotton plants collected from original author(s) and the source, provide a link to the Creative Commons licence, and indicate if changes were made. The images or other third party material in this article are included in the article's Creative Commons licence, unless indicated otherwise in a credit line to the material. If material is not included in the article's Creative Commons licence and your intended use is not permitted by statutory regulation or exceeds the permitted use, you will need to obtain permission directly from the copyright holder. To view a copy of this licence, visit http://creativecommons.org/licenses/by/4.0/. 
eight counties in Xinjiang, $42 \%$ of the $V$. dahliae isolates were defoliating pathotype, but the percentage varied widely among locations (Wagner et al. 2021). The results offered a better understanding of the epidemiology of Verticillium wilt in China. $V$. dahliae infection is complicated and involves a number of signaling pathways and biochemical substances (Fradin and Thomma 2006). As a result, there is no consensus on genetic mechanisms of cotton Verticillium wilt resistance across the world.

The long-term cotton production practice shows that mining and cloning resistant genes is an effective measure to control Verticillium wilt. Only the Gossypium barbadense has a higher resistance toward Verticillium wilt, but cultivating it on a large scale is difficult and obtaining a new breed with high resistance character by cross-breeding with the G. barbadense poses a major challenge (Cai et al. 2009). With the development of molecular biology and genetics of plant disease resistance, it is possible to carry out molecular breeding for disease resistance. However, a better understanding of the mechanisms of resistance are still required for improved plant resistance to Verticillium wilt. It is necessary to identify factors in the regulatory mechanism which provide the candidate genes for molecular breeding of cotton disease resistance. This paper will review the previous findings on cotton resistance to Verticillium wilt and integrate with the recent use of plant biotechnology in cotton resistance to Verticillium wilt to discuss the progress and ways forward on Verticillium wilt resistance.

\section{Genetic mapping based on different mapping populations}

A population in genetics generally refers to a species of an organism, a variety that contains variation or a group of varieties or even a specific generation of hybrids between individuals. Any genetic study will be incomplete without one or more populations with diverse genotypes. The main factors for effective and efficient quantitative trait loci (QTL) mapping are the selection of suitable mapping parents and the creation of appropriate population forms. Different mapping populations and molecular markers have been used in recent years to find QTLs with different genetic effects on 26 pairs of cotton chromosomes (Fig. 1). Possible candidate genes have been predicted in this field, and functional verification of some resistance genes has been performed.

\section{The parental mapping population}

The most commonly used genetic map is the parental mapping population. According to their characteristics, they can be divided into temporary groups and permanent groups. The transient population includes $F_{2}$ population, Backcross lines $(\mathrm{BC}) . \mathrm{F}_{2}$ population refers to the population obtained by random pollination of hybrid offspring at the beginning of $\mathrm{F}_{3}$ generation, and $\mathrm{BC}$ refers to the offspring crosses with either parent, continuous hybridization with one of the parents by multiple hybrids from the first generation onwards. Their most prominent feature is the rich genetic variation information in the offspring separation. The parental mapping population involves the choice of two parents with genetic

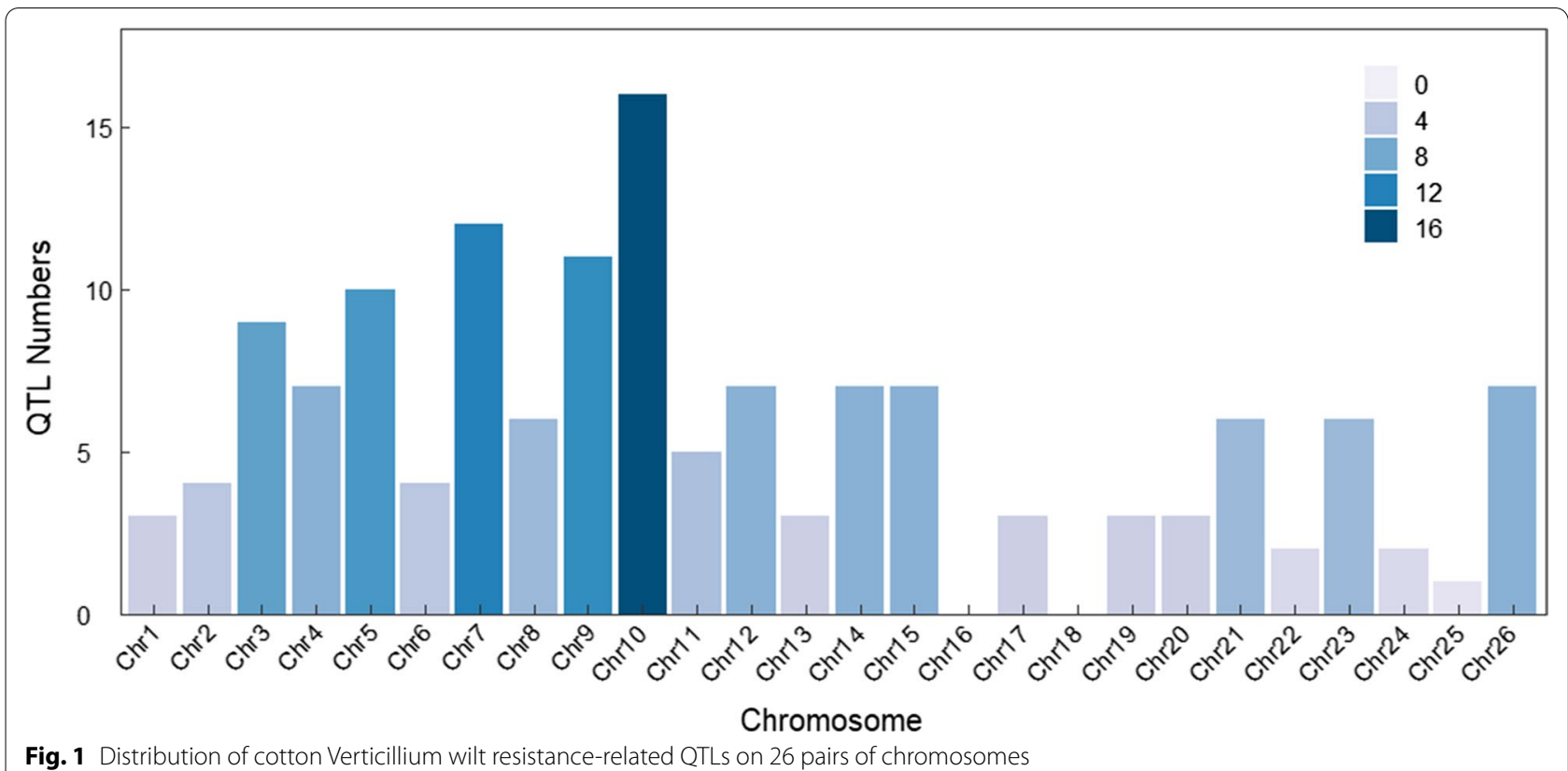

Fig. 1 Distribution of cotton Verticillium wilt resistance-related QTLs on 26 pairs of chromosomes 
differences to cross, in order to obtain more isolated chromosome regions which were suitable for the construction of genetic linkage map. The permanent group consists of Recombinant inbred lines (RIL), Double haploids (DH), Near-isogenic lines (NIL), Chromosome segment substitution lines (CSSLs). RIL is produced by multi-generation self-crossing of individuals in $\mathrm{F}_{2}$ population, and every line in the population is homozygous. NIL refers to a group of lines with the same or similar genetic background, but with differences in a particular trait or its genetic basis. DH is the diploid individual produced by artificial doubling of a diploid haploid gamete or by parthenogenetic/male development of a tetraploid gamete. CSSL is an overlapping chromosomal substitution population established in a recipient parent covering the entire genome of the donor parent, with one parent as the recipient and the other as the donor. The characteristics of permanent isolated populations are that multiple phenotypes can be identified at multiple points and at the same time, which improves the mapping accuracy.

Zhang et al. (2015) constructed an RIL population containing 146 lines and carried out a 4-year repeated identification of resistance to Verticillium wilt. A total of 10 resistance QTLs, 28 QTLs clusters, and 13 hot regions were identified for Verticillium wilt. Shi et al. (2016) constructed a BC population using high resistance Hai1 and high susceptibility CCRI36 as parents, a total of 48 QTLs related to Verticillium wilt resistance were identified with 37 QTLs showed additive effects. Rashid et al. (2021) selected 300 CSSLs from crossing Hai1 and CCRI36 and identified 40 QTLs related to Verticillium wilt which can explain $1.05 \% \sim 10.52 \%$ of the phenotypic variation. The results of this study establish a foundation for fine mapping and cloning of the resistance gene in the future.

Due to the limited number of recombination times of the parent mapping population, the low accuracy of the mapping and the genetic background of the parent population, the identification of the same mapped QTL loci or candidate genes in different populations is not highly efficient (Broman 2012).

\section{Artificially constructed multi-parental mapping population} At present, mapping population that were artificially constructed include Nested association mapping (NAM) population, Random-open-parent association mapping (ROAM) population and Multi-parent advanced generation inter-cross (MAGIC) population. Since the first two populations have not been involved in Verticillium wilt resistance studies in cotton, only the MAGIC population will be focused on. MAGIC population was constructed by polymerization and hybridization of multiple parents. The characteristics of this types of population is that it contains multiple parents, can carry more alleles, has no population structure, and can control the population size according to the needs of breeders.

MAGIC populations have been constructed in multiple crops including rice (Raghavan et al. 2017), wheat (Thepot et al. 2015), corn (Giraud et al. 2017), barley (Sannemann et al. 2015), faba beans (Sallam and Martsch 2015), sorghum (Ongom and Ejeta 2018) and other crops were mainly used for genetic analysis of agronomic traits. In the study of cotton phenotype, Islam et al. (2016) constructed a population containing 547 lines with 11 parents and polymerized hybridization for the first time, and analyzed the fiber quality traits of upland cotton. Relatively few studies have been conducted on mapping Verticillium wilt resistance loci in cotton based on multi-parent mapping. Zhang et al. (2020) constructed a MAGIC population containing 550 lines by a random mating of $11 \mathrm{G}$. hirsutum parents for five generations, followed by six generations of selfing, and identified 25 resistance related QTLs based on genome-wide association analysis of Verticillium wilt resistance in two replicated greenhouse tests.

\section{Natural population}

Natural populations are mainly derived from germplasm resources including bred varieties, landraces, wild species, genetic research materials etc., which can well represent the genetic characteristics of a species in a specific region or time period. Natural populations are rich in allelic variation and phenotypic variation, with high detection efficiency and relatively complete locus detection of controlling traits. The rich recombination information accumulated greatly improves the positioning accuracy even at the level of a single gene. Multiple alleles can be located in natural populations without the need of constructing a mapping population, which saves time and effort. In recent years, with the development of wholegenome sequencing, and large numbers of SNP markers, association analysis methods were used to detect genetic loci for natural populations.

Li et al. (2017b) used 299 germplasm resources and SNP polymorphism markers to perform a genomic association analysis of three environments. A total of 17 QTLs related to Verticillium wilt resistance were identified and 22 candidate genes were predicted. Gong et al. (2018) performed a genome-wide association study (GWAS) in 215 Chinese Gossypium arboreum accessions inoculated as seedlings with $V$. dahliae and identified 309 candidate loci involved in wilt resistance. The research newly-identified cluster of glutathione $S$-transferase genes GaGSTF9 was a positive regulator of Verticillium wilt. Resistance of 376 US G. hirsutum accessions to $V$. dahliae (defoliating pathotype) and virulent Fusarium oxysporum 
f. sp. vasinfectum race 4 (FOV4) was evaluated in four and two independent replicated greenhouse tests, respectively. A total of 15 and 13 QTL for Verticillium wilt and FOV4 resistances were anchored by 30 and 56 SNPs, respectively. Two QTL clusters on c16 (chr.16) and c19 (chr.19) were observed for both Verticillium wilt and FOV4 resistance, suggesting that these genomic regions may harbor genes in response to both diseases (Abdelraheem et al. 2020). Combining transcriptome, gene expression and functional validation analyses, Zhang et al. (2021) newly identified a Dt11 genomic region conferring strong Verticillium wilt resistance, and firstly revealed a GhLecRK-V.9 gene cluster playing a crucial role in Verticillium wilt resistance. Ma et al. (2021) generated two high-quality genomes of G. hirsutum cv. NDM8 and Gossypium barbadense acc. Pima 90 and identified large-scale structural variations in the two species and 1081 G. hirsutum accessions. They discovered that in many structural variations those for fiber quality and Verticillium wilt resistance are located mainly in the D-subgenome and those for yield mainly in the A-subgenome.

According to the genetic effects of disease-resistant QTLs in different populations, it is not difficult to find that the targeted population gradually shifts from the initial temporary segregation population to the permanent genetic one because the main agronomic traits in crop research are quantitative traits controlled by multiple genes. Genetic analysis is difficult to exclude QTLs or interactions between genes and to accurately study the interactions between QTLs.

Although the predecessors used different mapping populations and genetic markers to locate the QTLs related to Verticillium wilt resistance on the 26 pairs of chromosomes in cotton (Fig. 1), none has been applied to breeding practice. The main reasons are insufficient discovery of disease-resistant QTLs, a large amount of genetic information in a scattered state, excessive genetic distance from the target gene, and insufficient positioning accuracy (Palanga et al. 2017). In recent years, the completion of whole-genome sequencing of cotton based on natural mutation populations, multiple parent populations and the development of new molecular markers have laid the foundation for the use of molecular markers to assist cotton breeding against Verticillium wilt (Additional file 1: Table S1).

\section{Method on genetic mapping for quantitative traits}

After using the mapping population to obtain a genetic linkage map, we need to associate the phenotypic data with the QTL for analysis and locate the sites or markers linked to the target traits on the chromosomes. The main positioning methods are Single-marker analysis (SMA), Interval mapping (IM), Composite interval mapping (CIM), and Mixed-model based composite interval mapping (MCIM). The most commonly used models for genetic mapping of Verticillium wilt in cotton are CIM and MCIM.

The proposal of these methods provides a useful tool for the analysis of various complex genetic models. The genetic assumption of the CIM method was based on the mixed linear model in which the quantitative traits are controlled by multiple genes. It regards the population mean and the various genetic effects of QTL as fixed effects. It considers the effects of environment, QTL, environment and molecular markers as random ones. The combination of effect value estimation and positioning analysis can analyze the interaction effect between QTL and the environment but also improve the accuracy and the efficiency of mapping. Association analysis was conducted with the Genome-wide efficient mixed-model association (GEMMA) software package (Zhou and Stephens 2012). CottonSNP70K chip was used to identify the Verticillium wilt resistance QTLs by CIM method (Palanga et al. 2017). A total of 85630 cotton Verticillium wilt resistance trait-associated SNPs were used for GWAS with a compressed mixed linear model ( $\mathrm{Li}$ et al. 2017b). Approximately 3100 SSR markers were selected to identify Verticillium wilt resistance by CIM (Zhao et al. 2018). For mixed-linear-model analysis, Zhang et al. (2021) used the following equation: $y=X \alpha+S \beta$ $+\mathrm{K} \mu+\mathrm{e}$, identified D11 genomic region associated with cotton Verticillium wilt resistance.

In summary, QTL mapping methods range from single marker to multiple markers, and from single trait to complex traits. The genetic effects of multiple traits are considered together, and the methods are becoming more and more accurate. Since the environment has a great influence on quantitative traits and directly affects the effect of QTL positioning, the positioning analysis starts from the marker itself and considers the principle of interaction with the environment to achieve more effective and practical applications.

\section{Genetic analysis methods for quantitative trait loci}

Crops have many agronomic traits which can be divided into qualitative traits and quantitative traits. Qualitative traits are characterized by discontinuous variation, they are easy to identify, stable phenotype, controlled by one or a few pairs of genes and relatively easy to study. However, quantitative traits show continuous variation, then are generally controlled by multiple minor genes and have complex signal network regulation pathways. Quantitative traits are easily affected by the genotype, 
environment and the interaction of environment. Their phenotypic variation is rich and the research is relatively difficult. Primary breeding targets of G. hirsutum such as yield, maturity, Verticillium wilt resistance and fiber quality are all quantitative traits. The current genetic methods used to analyze quantitative traits mainly include QTL mapping based on linkage balance and association analysis based on linkage disequilibrium (LD).

\section{Linkage analysis}

Linkage analysis refers to linkage mapping or linkage QTL mapping. It requires the construction of segregating population with statistical exchange values and recombination status. The segregating populations used in linkage analysis are constructed based on the parents. The derived populations of the parents have undergone a certain reorganization which effectively breaks the linkage to a certain extent. It has advantages in preliminary positioning and fine positioning but also has some shortcomings. Due to the limited number of recombination, the positioning accuracy is low; the allelic variation only comes from two donor parents, the detection efficiency is low, the genes or loci found are limited and the whole genome cannot be covered. The detected loci are affected by the genetic background of the parents. The repeatability of points among different groups is not high and unstable.

\section{Association analysis}

Association analysis also known as LD mapping is a method of associating target traits with related markers based on LD. According to the LD decay, the candidate interval and even the candidate gene of the target trait can be locked (Gaut and Long 2003). Using this method, alleles closely related to phenotypic variation can be directly identified and the target traits can be quickly and finely mapped. Association analysis was first applied to the study of human genetics and its application to plants has only recently begun. Thornsberry et al. (2001) successfully introduced association analysis into plants for the first time. The application of the association analysis in the study of cotton quantitative traits started late compared with QTL linkage mapping (Abdurakhmonov et al. 2008). So far, there are few reports on cotton Verticillium wilt resistance using association analysis.

Genome-wide association analysis based on LD uses the phenotypic variation of natural populations to overcome the limitations of parents and obtain QTL maps with higher resolution which has become a powerful tool for plant genetic map construction (Ma et al. 2018). With the development of a large number of SNP markers and the rapid development of bioinformatics, the association analysis method has become one of the latest research hotspots in international plant genomics (Dehghan 2018). At the same time, the QTL location method based on association analysis has formed a relatively complete technical system. This method has been widely used in the location of some quantitative traits in corn ( $\mathrm{Li}$ et al. 2019a), soybean (Wang et al. 2018a), wheat (Juliana et al. 2019), sorghum (Tao et al. 2020), rice (Takatsuji and Hayashi 2017), cotton (Li et al. 2019b) and other crops. In cotton, it was first used in the research of yield and fiber quality related traits (Abdurakhmonov et al. 2008); recently, it has shown obvious advantages in the research of the fine positioning of cotton resistance-related molecular markers and the genetic mechanism of some complex quantitative traits.

Linkage analysis and association analysis both play an important role in quantitative trait research. They have obvious complementarity in the accuracy and breadth of QTL mapping, the amount of information provided and statistical analysis methods. Linkage analysis can initially locate loci controlling target traits. The location of the locus and association analysis can quickly locate the target gene and provide a large amount of information for a specific candidate gene to verify its function. Combining the advantages of linkage analysis and association analysis, dissecting quantitative traits vertically and horizontally will speed up the identification, isolation and cloning of quantitative trait genes and provide a better understanding of the genetic and molecular biological basis of quantitative traits and the basis of crop quantitative traits. Genetic improvement provides new opportunities.

\section{Inheritance of resistance to Verticillium wilt in cotton and cloning of disease resistance genes Pathogenicity of Verticillium dahliae and inheritance of resistance to Verticillium wilt in cotton}

There are many factors involved in the inheritance of cotton resistance to Verticillium wilt due to the differences in the disease resistance of the parent materials, the pathogenicity of $V$. dahliae, the identification method, the identification environment, the amount of inoculation, the classification index and the statistical method of the disease rating, etc. The results of the researches are different and there is no unified conclusion. In an environment with controlled conditions, in a greenhouse or culture room and inoculated with a single strain, the resistance of cotton to Verticillium wilt is controlled by a single gene or a few major genes (Bolek et al. 2005).

The polyketo synthase gene VdPKS1 was found to form melanin-deficient albino sclerotia without affecting fungal colonization in host tissues but significantly reducing disease severity. Melanin synthesis mediated by VdPKS1 inhibits ethylene biosynthesis and affects the expression 
of a series of genes associated with micronucleus formation and pathogenesis. The results indicated that VdPKS1 had important significance for virulence and development of $V$. dahliae (Zhang et al. 2017b). In this study, they found an effector molecule VdSCP7 with a special mechanism of action. The virulence of VdSCP7 knockout mutant was significantly enhanced in cotton hosts, indicating that there may be a potential resistance gene in cotton hosts to recognize VdSCP7 in the fungus, and activate plant immunity (Zhang et al. 2017a). VdRGS1 gene in $V$. dahliae Vd8 was knocked-out by Agrobacterium-mediated method and complement mutant function and phenotype analysis showed that VdRGS1 regulated the spore formation and mycelium growth of microsclerotia of $V$. dahliae, and played an important role in host infection of $V$. dahliae (Xu et al. 2018). As the first line of defense of plants, reactive oxygen species (ROS) on the one hand initiates the transmission of immune signals, and on the other hand directly inhibits the growth of pathogenic fungi. These findings suggest that soil-borne pathogenic fungi respond to host ROS stress through chromatin remodeling, and repair fungal DNA damage caused by ROS, which further advances the understanding of the pathogenic mechanism of $V$. dahliae and provides an experimental basis for the study of chromatin remodeling complexes of other pathogenic fungus (Wang et al. 2020a, b).

However, the resistance of $G$. hirsutum to $V$. dahliae in the field or multi-pathogen environment is expressed as a quantitative trait inheritance (Wang et al. 2008). In response to the latter conclusion, many studies have been carried out around the world where the disease resistance breeding based on molecular markers provides a new perspective for the analysis of cotton Verticillium wilt resistance inheritance.

\section{Mining and cloning of genes as related to the resistance to Verticillium wilt in cotton}

When pathogens invade the plant, the plant will activate its own immune system and regulate the expression of many genes at the transcriptional level to participate in the plant disease-resistance response.

The earliest researchers cloned tomato genes $V e 1$ and $\mathrm{Ve} 2$ that are resistant to $V$. dahliae and encode cell surface receptor-like protein (Kawchuk et al. 2001). After the $V e 1$ gene was transformed into tobacco and cotton, respectively, it was found that the transgenic plants had increased the resistance to V.dahliae 'V991' (Fradin et al. 2009). Further studies have shown that Ve1-mediated resistance to $V$. dahliae is race-specific, and Ve1 can only recognize the non-toxic protein Ave1 in the physiological race1 (non-defoliating pathotype) of $V$. dahliae (Castroverde et al. 2016).
Plant hormones, as signal substances, not only regulate plant growth, development, morphogenesis, reproduction, but also participate in plant response to biological or abiotic stress. Cotton transcription factor GbWRKY1 can directly regulate the jasmonic acid (JA) signal pathway and negatively regulate the expression of JAZ1. Overexpression of GbWRKY1 in cotton and Arabidopsis attenuates the resistance of transgenic plants to Verticillium wilt (Li et al. 2014). The cotton GhSSN gene encodes cytochrome P450 oxidases, and cotton seedlings show constitutive JA synthesis and activation of signal pathways after inhibiting the expression of this gene (Sun et al. 2014). GbERF1 and GbERF2 cloned from G. barbadense induced the express of ethylene signaling pathway related genes. GbERF1-like has similar expression characteristics to GbERF1 and GbERF2. Overexpression of this gene in cotton and Arabidopsis can speed up the expression of genes related to lignin synthesis and enhance resistance to Verticillium wilt (Guo et al. 2016). A new cotton germplasm with high resistance to Verticillium wilt was successfully developed in response to salicylic acid (SA) and JA signaling pathways by extracellular induction and expression of antifungal protein GAFP4. Transgenic plants of $G A D$, a gene responsible for GABA synthesis in $A$ thaliana, are more susceptible to disease when they lack GABA and overaccumulation of GABA inhibits plant growth (Wang et al. 2020a, b). Overexpression of the GbMPK3 gene isolated from the root system of $G$. barbadense increased the resistance of cotton to Verticillium wilt and activated the SA signaling pathway, which played a major role in the defense response of cotton against Verticillium wilt (Long et al. 2020). The APETALA2/ ETHYLENE RESPONSIVE FACTOR gene GhTINY2 overexpression in cotton and $A$. thaliana enhanced tolerance to $V$. dahliae, while knockdown of expression increased the susceptibility of cotton to the pathogen. GhTINY2 was found to promote SA accumulation and SA signaling transduction by directly activating expression of WRKY51 (Xiao et al. 2021b).

Plants can produce many primary and secondary metabolites to maintain normal growth and development and resist environmental stress. To elucidate the function of G. hirsutum DIR genes in lignification, GhDIR1 encoding putative dirigent proteins that was preferentially accumulated in cotton hypocotyls, overexpression of GhDIR1 gene resulted in an increase in lignin content in transgenic cotton plants. Compared with that of wild type, the transgenic cotton plants displayed more tolerance to the infection of $V$. dahliae (Shi et al. 2012). In the study of the upstream regulatory factors and regulation modes of gossypol metabolism, the expression of GbFPS, GbCAD1 and WRKY1 in cotton plants was upregulated by SA and methyl jasmonate (MeJA), which 
are plant hormones related to disease resistance (Gao et al. 2013). The tomato Ve homologous gene Gbvdr3 overexpression level in cotton, the content of hydrogen peroxide and callose increased, the expression level of disease course related genes increased, which could significantly improve the resistance of plants to verticillium wilt (Chen et al. 2016). GhLac1 gene cloned from cotton protoplasts can promote lignin synthesis and enhance broad-spectrum resistance of cotton. Inhibition of GhLac1 expression can promote the synthesis of secondary flavonoid metabolites and JA of cotton and enhance the resistance of transgenic materials to $V$. dahliae and cotton bollworm (Hu et al. 2019). Tryptophan synthesisrelated genes GbTSA1 and GbTSB1 may be involved in the broad-spectrum response of plant disease resistance signal pathways or immune regulation to fungal infection (Xu et al. 2014). Further studies showed that the suppression of the expression of GbTSA1 and GbTRP1 genes related to tryptophan synthesis could significantly activate the immune response and enhance the resistance of cotton to Verticillium wilt (Miao et al. 2019). G. hirsutum ribosomal protein L18A gene GhARPL18A-6 was highly induced in G. hirsutum after inoculation with $V$. dahliae. Knock-down of GhARPL18A-6 increased lignin deposition, an enhanced ROS burst, and phenylpropanoid biosynthesis defense response pathways all contributed to a decrease in colonization by $V$. dahliae (Zhang et al. 2019b). GhMYB4, a negative regulator of lignin synthesis identified in cotton, reduces lignin deposition and enhances resistance to $V$. dahliae (Xiao et al. 2021a).

The mining of cotton Verticillium wilt resistance genes and the verification of their functions helped for the analysis of the molecular mechanism of cotton disease resistance, the mining of resistant germplasm resources and the selection and breeding of new disease-resistant varieties. The following figure shows the functional genes of resistance to Verticillium wilt in cotton and the major signal regulation networks they involved (Fig. 2).

\section{Functional verification of disease resistance genes Gene editing technology}

Gene editing technology uses artificial engineered and modified nucleases (ZFN) to modify the genome and its transcription products of organisms to achieve the purpose of changing the target genes, regulatory element sequences, expression levels or functions (Komor et al. 2016). In recent years, new technologies represented by the Clustered regularly interspaced short palindromic repeats (CRISPR)/CRISPR associated Protein 9 (CRICPR/Cas9) system have been widely used in the genetic improvement of crops.

Some of the main agronomic traits of crops are determined by point mutations or single base changes in a certain gene. Wheat belongs to allopolyploid crop and its gene family is large, so it is difficult to use traditional breeding for crop improvement. A mutant with three TaMLO homologous genes knocked out simultaneously was obtained by using CRICPR/Cas9 technique. The mutant showed resistance to wheat powdery mildew (Wang et al. 2014). The tagasr 7 mutant significantly increased the grain weight and tadep 1 mutant significantly decreased the plant height (Zhang et al. 2016). For the first time, a single base editing system based on gene editing was used to replace the base of nrT1.1b and DELLA family SLR1 to improve nitrogen use efficiency in rice (Li et al. 2017a). Maize ARGOS8 gene is a negative regulator of ethylene response in plants. By using CRICPR/Cas9 editing technique, ARGOS8 promoter was replaced with GOS2 promoter to reduce the effect of drought stress on grain yield of the mutant plant (Shi et al. 2017). The knockout of $Z m N C 1 / Z m H K T 1$ QTL for salt tolerance in maize increased the tolerance to salt stress (Zhang et al. 2018b).

CRISPR/Cas9 knockout of two GhARG homologous genes in upland cotton inhibited arginase activity but activated nitric oxide synthase activity, which induced arginine synthesis of more nitric oxide (NO) and promoted lateral root formation (Wang et al. 2017). After the endogenous gene GhCLA1 was edited by CRISPR/ Cas9, $75 \%$ of the $T_{0}$ plants showed albino seedling phenotype (Wang et al. 2018b). The transgene free mutants created based on genome editing technology have high resistance to cotton Verticillium wilt and provide excellent germplasm resources for improving cotton Verticillium wilt resistance. Meanwhile, this study also elucidates for the first time the rules and identification methods of cotton genes edited simultaneously in two subgroups A and D (Zhang et al. 2018c). GhBE3 gene editing system was developed in cotton to produce single base mutation and improve the efficiency of transgenic plants (Qin et al. 2020). Temperature-sensitive CRISPR/LbCpf1 (LbCas12a)-mediated genome editing system creates homozygous, gossypol free, transgene free cotton materials, providing new germplasm resources for cotton molecular breeding (Li et al. 2021).

Because gene editing technology has the advantages of simple operation, high efficiency and low cost, it has great potential and broad application prospects in crop genetic research. Although its application in cotton is currently limited, its successful examples in major crops such as wheat, rice and corn provide theoretical foundation and technical support for its later application in cotton disease resistance breeding. 


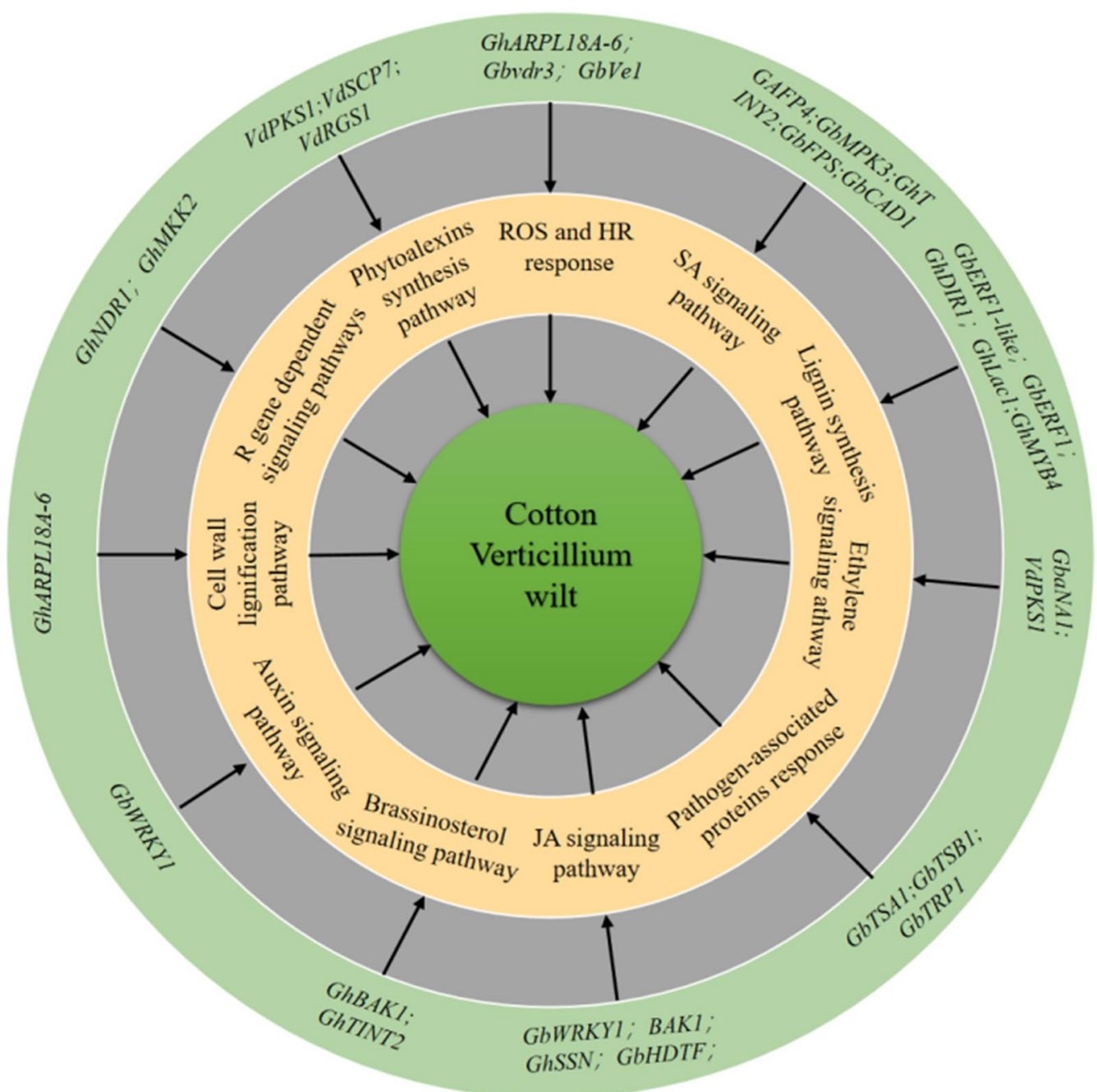

Fig. 2 Functional genes related to cotton resistance to Verticillium wilt

\section{Virus-induced gene silencing technology}

Virus-induced gene silencing (VIGS) is a natural mechanism for plants to resist virus infection. It is a phenomenon of post-transcriptional gene silencing, which is widespread in plants. VIGS has been widely used in the research of plant gene function. With the completion of cotton genome sequencing, VIGS technology has played an important role in the mining and functional research of cotton Verticillium wilt related genes.

Cotton plants with suppressed KATANIN expression produced shorter fibers and elevated weight ratio of seed oil to endosperm. By contrast, silencing of WRINKLED1 expression resulted in increased fiber length but reduced oil seed content, suggesting the possibility to increase fiber length by repartitioning carbon flow $(\mathrm{Qu}$ et al.
2012). Further, Tobacco rattle virus induced gene silencing (TRV-VIGS) was used to investigate the function of GhBI-1 (cotton Bax inhibitor-1) gene in response to salt stress and the result demonstrated that GhBI-1 might play a protective role under salt stress by suppressing stress-induced cell death in cotton (Zhang et al. 2018a). This result showed that the TRV-VIGS system can be used for rapid functional analysis of genes involved in cotton fiber development. The resistance of cotton to Verticillium wilt was significantly reduced by silencing GhNDR1, GhMKK2, and GhBAK1, homologs of NDR1, $M K K 2$, and $B A K 1$ of the Ve1 signaling pathway in $A$. thaliana through VIGS (Gao et al. 2011).

The advantage of the VIGS technology applicated in cotton is obvious. No genetic transformation is needed 
and the effect of gene silencing can be achieved in a short time. Moreover, VIGS technology is independent of cotton genotype and the silencing efficiency is high. In addition, VIGS can simultaneously silences multiple target genes on the same cotton plant. VIGS technology also has its shortcomings. Its silencing of target genes cannot be inherited to the next generation, and only occurs in contemporary plants; and the efficiency of gene silencing is inconsistent. With the in-depth exploration of VIGS technology, the development of more VIGS vectors and the improvement of inoculation technology, it is predicted that VIGS technology will play a greater role in the research of cotton functional genes.

\section{Summary and prospect}

In recent decades, with the vigorous promotion of insectresistant cotton in China and the selection and successful breeding of Fusarium wilt resistant varieties, cotton Verticillium wilt has become the most important disease restricting cotton production. Verticillium dahliae has co-evolved with plants (Chen et al. 2021). Therefore, the key to durable and effective cotton disease resistance lies in the interaction mechanism between cotton and $V$. dahliae. Predecessors analyzed the relationship between cotton and $V$. dahliae from the aspects of cotton's tissue structure resistance, physiological and biochemical resistance, R-gene-mediated resistance, hormone-mediated disease resistance signal pathways and their interactions. There is no clear understanding currently of the mechanisms involved with interactions, and further research is needed. With the completion of cotton whole-genome sequencing and the development of high-throughput molecular markers, the quantitative trait positioning based on molecular markers makes it possible to finely locate the site of gene resistant to Verticillium wilt. In addition, predecessors used genetic engineering technology to clone disease-related genes. VIGS and host-induced gene silencing (HIGS) suppresses the growth or pathogenicity of pathogens in host plants by silencing key genes of pathogens to achieve the purpose of reducing plant diseases. In summary, for the control of cotton Verticillium wilt, breeding and planting diseaseresistant varieties with competitive yield and fiber properties is a necessity. The progress of genetically modified cotton breeding germplasm is slow, and it is difficult for the selected varieties to adapt to different ecological environments. Therefore, the combination of traditional breeding and modern biotechnology will be necessary to accelerate the process of disease resistance breeding in the future.

\section{Supplementary Information}

The online version contains supplementary material available at https://doi. org/10.1186/s42397-021-00109-0.

Additional file 1: Table S1. Cotton Verticillium wilt resistance-related QTLs has been reported previously.

\section{Acknowledgements}

We would like to thank the anonymous reviewers for their valuable comments and helpful suggestions which help to improve the manuscript.

\section{Authors' contributions}

Nie XH, Zhu LF and Pan ZY designed the experiments. Jibril AN, Liu SM and Han P performed the experiments. Aini N wrote the main manuscript and prepared all figures. Han P performed data analysis. Nie XH, Pan ZY, Zhu LF and Liu SM revised and polished the manuscript. All authors contributed in the interpretation of results, read and approved the final manuscript.

\section{Funding}

This work was supported by the National Natural Science Foundation of China (31760402), Young and Middle-aged Science and Technology Leading Talents of Xinjiang Production and Construction Corps (2019CB027), Science and Technology Innovation Talent Plan of Xinjiang Production and Construction Corps (2021CB028), National Natural Funds-Xinjiang Joint Fund (U1703231).

\section{Availability of data and materials}

The datasets used and analyzed during the current study are available from the corresponding author on reasonable request.

\section{Declarations}

Ethics approval and consent to participate

Not applicable.

Consent for publication

All Authors have provided ethical approval and consent to participate as well as consent for publication.

\section{Competing interests}

The authors have declared that no competing interests exist.

\section{Author details}

${ }^{1}$ Key Laboratory of Oasis Ecology Agricultural of Xinjiang Bingtuan, Agricultural College, Shihezi University, Shihezi 832003, Xinjiang, China. ${ }^{2}$ National Key Laboratory of Crop Genetic Improvement, College of Plant Sciences and Technology, Huazhong Agricultural University, Wuhan 430070, Hubei, China.

Received: 7 September 2021 Accepted: 6 December 2021

Published online: 20 January 2022

\footnotetext{
References

Abdelraheem A, Elassbli H, Zhu Y, et al. A genome-wide association study uncovers consistent quantitative trait loci for resistance to Verticillium wilt and Fusarium wilt race 4 in the US Upland cotton. Theor Appl Genet. 2020;133:563-77. https://doi.org/10.1007/s00122-019-03487-X.

Abdurakhmonov IY, Kohel RJ, Yu JZ, et al. Molecular diversity and association mapping of fiber quality traits in exotic G. hirsutum L. germplasm. Genomics. 2008;92:478-87. https://doi.org/10.1016/j.ygeno.2008.07.013.

Bolek Y, El-Zik KM, Pepper AE, et al. Mapping of verticillium wilt resistance genes in cotton. Plant Sci. 2005;168:1581-90. https://doi.org/10.1016/j. plantsci.2005.02.008.

Broman KW. Genotype probabilities at intermediate generations in the construction of recombinant inbred lines. Genetics. 2012;190(2):403-12. https://doi.org/10.1534/genetics.111.132647.
} 
Cai YF, He XH, Mo JC, et al. Molecular research and genetic engineering of resistance to Verticillium wilt in cotton: a review. Afr J Biotech. 2009;8:7363-72. https://doi.org/10.5897/AJB2009.000-9571.

Castroverde CDM, Nazar RN, Robb J. Verticillium Ave1 effector induces tomato defense gene expression independent of Ve1 protein. Plant Signal Behav. 2016;1 1:e1245254. https://doi.org/10.1080/15592324.2016.1245254.

Chen TZ, Kan JL, Yang YW, et al. A Ve homologous gene from Gossypium barbadense, Gbvdr3, enhances the defense response against Verticillium dahliae. Plant Physiol Biochem. 2016;98:101-11. https://doi.org/10.1016/j. plaphy.2015.11.015

Chen JY, Klosterman SJ, Hu XP, et al. Key insights and research prospects at the dawn of the population genomics era for Verticillium dahliae. Annu Rev Phytopathol. 2021;59:31-51. https://doi.org/10.1146/annur ev-phyto-020620-121925.

Dehghan A. Genome-wide association studies. In: Evangelou E, editor. Genetic epidemiology. Methods in Molecular Biology, vol. 1793. New York, NY: Humana Press; 2018. p. 37-49. https://doi.org/10.1007/ 978-1-4939-7868-7 4

Fradin EF, Thomma BPHJ. Physiology and molecular aspects of Verticillium wilt diseases caused by V. dahliae and V. albo-atrum. Mol Plant Pathol. 2006;7:71-86. https://doi.org/10.1111/j.1364-3703.2006.00323.X.

Fradin EF, Zhang Z, Juarez Ayala JC, et al. Genetic dissection of Verticillium wilt resistance mediated by tomato Ve1. Plant Physiol. 2009;150:320-32. https://doi.org/10.1104/pp.109.136762.

Gao XQ, Wheeler T, Li ZH, et al. Silencing GhNDR1 and GhMKK2 compromises cotton resistance to Verticillium wilt. Plant J. 2011;66(2):293-305. https:// doi.org/10.1111/j.1365-313X.2011.04491.X.

Gao W, Long L, Zhu LF, et al. Proteomic and virus-induced gene silencing (VIGS) analyses reveal that gossypol, brassinosteroids, and jasmonic acid contribute to the resistance of cotton to Verticillium dahliae. Mol Cell Proteomics. 2013;12:3690-703. https://doi.org/10.1074/mcp.M113.031013.

Gaut BS, Long AD. The lowdown on linkage disequilibrium. Plant Cell. 2003;15:1502-6. https://doi.org/10.1105/tpc.150730.

Giraud H, Bauland C, Falque M, et al. Linkage analysis and association mapping QTL detection models for hybrids between multiparental population from two heterotic groups: application to biomass production in maize (Zea mays L.). G3 Genes Genomes Genetics. 2017;7:3649-57. https://doi. org/10.1534/g3.117.300121.

Gong Q, Yang ZE, Chen EY, et al. A phi-class glutathione S-transferase gene for Verticillium wilt resistance in Gossypium arboreum identified in a genome-wide association study. Plant Cell Physiol. 2018;59:275-89. https://doi.org/10.1093/pcp/pcx180.

Guo WF, Jin L, Miao YH, et al. An ethylene response-related factor, GbERF1like, from Gossypium barbadense improves resistance to Verticillium dahliae via activating lignin synthesis. Plant Mol Biol. 2016;91:305-18. https:// doi.org/10.1007/s11103-016-0467-6.

Hu XP, Gurung S, Short DPG, et al. Nondefoliating and defoliating strains from cotton correlate with races 1 and 2 of Verticillium dahliae. Plant Dis. 2015;99:1713-20. https://doi.org/10.1094/pdis-03-15-0261-re.

$\mathrm{Hu} Q$, Xiao SH, Guan QQ, et al. The laccase gene GhLac1 modulates fiber initiation and elongation by coordinating Jasmonic acid and flavonoid metabolism. Crop J. 2019;8(4):522-33. https://doi.org/10.1016/j.cj.2019. 11.006 .

Islam MS, Thyssen GN, Jenkins JN, et al. A MAGIC population-based genomewide association study reveals functional association of GhRBB1_A07 gene with superior fiber quality in cotton. BMC Genomics. 2016;17:903. https://doi.org/10.1186/s12864-016-3249-2.

Juliana P, Poland J, Huerta-Espino J, et al. Improving grain yield, stress resilience and quality of bread wheat using large-scale genomics. Nat Genetics, 2019:51:1530-9. https://doi.org/10.1038/s41588-019-0496-6.

Kawchuk LM, Hachey J, Lynch DR, et al. Tomato Ve disease resistance genes encode cell surface-like receptors. Proc Natl Acad Sci USA. 2001;98:65115. https://doi.org/10.1073/pnas.091114198.

Komor AC, Kim YB, Packer MS, et al. Programmable editing of a target base in genomic DNA without double-stranded DNA cleavage. Nature. 2016:533:420-424. https://doi.org/10.1038/nature17946.

Li C, He X, Luo XY, et al. Cotton WRKY1 mediates the plant defense-todevelopment transition during infection of cotton by Verticillium dahliae by activating JASMONATE ZIM-DOMAIN1 expression. Plant Physiol. 2014;166:2179-94. https://doi.org/10.1104/pp.114.246694.
Li JY, Sun YW, Du JL, et al. Generation of targeted point mutations in rice by a modified CRISPR/Cas9 system. Mol Plant. 2017a;10:526. https://doi.org/ 10.1016/j.molp.2016.12.001.

Li TG, Ma XF, Li NY, et al. Genome-wide association study discovered candidate genes of Verticillium wilt resistance in upland cotton (Gossypium hirsutum L.). Plant Biotechnol J. 2017b;15:1520-32. https://doi.org/10.1111/pbi. 12734 .

Li N, Lin B, Wang H, et al. Natural variation in ZmFBL41 confers banded leaf and sheath blight resistance in maize. Nat Genetics. 2019a;51:1540-8. https:// doi.org/10.1038/s41588-019-0503-y.

Li JY, Wang MJ, Li YJ, et al. Multi-omics analyses reveal epigenomics basis for cotton somatic embryogenesis through successive regeneration acclimation process. Plant Biotechnol J. 2019b;17:435-50. https://doi.org/10. 1111/pbi.12988.

Li B, Liang SJ, Alariqi M, et al. The application of temperature sensitivity CRISPR/ LbCpf1 (LbCas12a) mediated genome editing in allotetraploid cotton (G. hirsutum) and creation of nontransgenic, gossypol-free cotton. Plant Biotechnol J. 2021;19:221-3. https://doi.org/10.1111/pbi.13470.

Long L, XU FC, Zhao JR, et al. GbMPK3 overexpression increases cotton sensitivity to Verticillium dahliae by regulating salicylic acid signaling. Plant Sci. 2020;292:110374. https://doi.org/10.1016/j.plantsci.2019.110374.

Ma ZY, He SP, Wang XF, et al. Resequencing a core collection of upland cotton identifies genomic variation and loci influencing fiber quality and yield. Nat Genetics. 2018:50:803-13. https://doi.org/10.1038/ s41588-018-0119-7.

Ma ZY, Zhang Y, Wu LQ, et al. High-quality genome assembly and resequencing of modern cotton cultivars provide resources for crop improvement. Nat Genet. 2021;53:1385-91. https://doi.org/10.1038/ s41588-021-00910-2.

Miao YH, Xu L, He X, et al. Suppression of tryptophan synthase activates cotton immunity by triggering cell death via promoting SA synthesis. Plant J. 2019;98:329-45. https://doi.org/10.1111/tpj.14222.

Ongom PO, Ejeta G. Mating design and genetic structure of a multi-parent advanced generation intercross (MAGIC) population of sorghum (Sorghum bicolor (L.) Moench). G3 Genes Genomes Genetics. 2018;8:331-41. https://doi.org/10.1534/g3.117.300248.

Palanga KK, Jamshed M, Rashid MHO, et al. Quantitative trait locus mapping for Verticillium wilt resistance in an upland cotton recombinant inbred line using SNP-based high density genetic map. Front Plant Sci. 2017:8:382. https://doi.org/10.3389/fpls.2017.00382.

Qin L, Li JY, Wang QQ, et al. High-efficient and precise base editing of C.G to T.A in the allotetraploid cotton (Gossypium hirsutum) genome using a modified CRISPR/Cas9 system. Plant Biotechnol J. 2020;18:45-56. https:// doi.org/10.1111/pbi.13168.

Qu J, Ye J, Geng YF, et al. Dissecting functions of KATANIN and WRINKLED1 in cotton fiber development by virus-induced gene silencing. Plant Physiol. 2012;160:738-48. https://doi.org/10.1104/pp.112.198564.

Raghavan C, Mauleon R, Lacorte V, et al. Approaches in characterizing genetic structure and mapping in a rice multiparental population. G3 Genes Genomes Genetics. 2017;7:1721-30. https://doi.org/10.1534/g3.117. 042101.

Rashid MH, Li PT, Chen TT, et al. Genome-wide quantitative trait loci mapping on Verticillium wilt resistance in 300 chromosome segment substitution lines from Gossypium hirsutum × Gossypium barbadense. G3 Genes Genomes Genetics. 2021;11(5):jkab027. https://doi.org/10.1093/g3jou rnal/jkab027.

Sallam A, Martsch R. Association mapping for frost tolerance using multiparent advanced generation inter-cross (MAGIC) population in faba bean (Vicia faba L.). Genetica. 2015;143:501-14. https://doi.org/10.1007/ s10709-015-9848-z.

Sannemann W, Huang BE, Mathew B, Leon J. Multi-parent advanced generation inter-cross in barley: high-resolution quantitative trait locus mapping for flowering time as a proof of concept. Mol Breeding. 2015;35:86. https://doi.org/10.1007/s11032-015-0284-7.

Shi HY, Liu ZH, Zhu L, et al. Overexpression of cotton (Gossypium hirsutum) dirigent1 gene enhances lignification that blocks the spread of Verticillium dahliae. Acta Biochim Biophys Sin. 2012;44:555-64. https://doi.org/ 10.1093/abbs/gms035.

Shi YZ, Zhang BC, Liu AY, et al. Quantitative trait loci analysis of Verticillium wilt resistance in interspecific backcross populations of Gossypium hirsutum 
× Gossypium barbadense. BMC Genomics. 2016;17:877. https://doi.org/10. 1186/s12864-016-3128-X.

Shi JR, Gao HR, Wang HY, et al. ARGOS8 variants generated by CRISPR-Cas9 improve maize grain yield under field drought stress conditions. Plant Biotechnol J. 2017;15(2):207-16. https://doi.org/10.1111/pbi.12603.

Song RR, Li JP, Xie CJ, et al. An overview of the molecular genetics of plant resistance to the Verticillium wilt pathogen Verticillium dahliae. Int J Mol Sci. 2020;21(3):1120. https://doi.org/10.3390/ijms21031120.

Sun LQ, Zhu LF, Xu L, et al. Cotton cytochrome P450 CYP82D regulates systemic cell death by modulating the octadecanoid pathway. Nat Commun. 2014;5:5372. https://doi.org/10.1038/ncomms6372.

Takatsuji H, Hayashi N. Broad-spectrum blast resistance: harnessing a natural allele of a transcription factor in rice. Mol Plant. 2017;10:1144-6. https:// doi.org/10.1016/j.molp.2017.08.005.

Tao YF, Zhao XR, Wang XM, et al. Large-scale GWAS in sorghum reveals common genetic control of grain size among cereals. Plant Biotechnol J. 2020;18:1093-105. https://doi.org/10.1111/pbi.13284.

Thepot S, Restoux G, Goldringer I, et al. Efficiently tracking selection in a multiparental population: the case of earliness in wheat. Genetics. 2015;199:609-21. https://doi.org/10.1534/genetics.114.169995.

Thornsberry JM, Goodman MM, Doebley J, et al. Dwarf8 polymorphisms associate with variation in flowering time. Nat Genet. 2001;28:286-9. https:// doi.org/10.1038/90135.

Wagner TA, Gu AX, Duke SE, et al. Genetic diversity and pathogenicity of Verticillium dahliae isolates and their co-occurrence with Fusarium oxysporum f. sp. vasinfectum causing cotton wilt in Xinjiang, China. Plant Dis. 2021;105:978-85. https://doi.org/10.1094/pdis-09-20-2038-re.

Wang HM, Lin ZX, Zhang XL, et al. Mapping and quantitative trait loci analysis of Verticillium wilt resistance genes in cotton. J Integr Plant Biol. 2008;50:174-82. https://doi.org/10.1111/j.1744-7909.2007.00612.x.

Wang YP, Cheng X, Shan QW, et al. Simultaneous editing of three homoeoalleles in hexaploid bread wheat confers heritable resistance to powdery mildew. Nat Biotechnol. 2014;32:947-51. https://doi.org/10.1038/nbt. 2969.

Wang YL, Meng ZG, Liang CZ, et al. Increased lateral root formation by CRISPR/ Cas9-mediated editing of arginase genes in cotton. Sci China Life Sci. 2017;60:524-7. https://doi.org/10.1007/s11427-017-9031-y.

Wang M, Li WZ, Fang C, et al. Parallel selection on a dormancy gene during domestication of crops from multiple families. Nat Genetics. 2018a;50:1435-41. https://doi.org/10.1038/s41588-018-0229-2.

Wang PC, Zhang J, Sun L, et al. High efficient multisites genome editing in allotetraploid cotton (Gossypium hirsutum) using CRISPR/Cas9 system. Plant Biotechnol J. 2018b;16:137-50. https://doi.org/10.1111/pbi.12755.

Wang S, Wu XM, Liu CH, et al. Verticillium dahliae chromatin remodeling facilitates the DNA damage repair in response to plant ROS stress. PLoS Pathog. 2020a;16(4):e1008481. https://doi.org/10.1371/journal.ppat.1008481.

Wang YQ, Liang CZ, Wu SJ, et al. Vascular-specific expression of Gastrodia antifungal protein gene significantly enhanced cotton Verticillium wilt resistance. Plant Biotechnol J. 2020b;18:1498-500. https://doi.org/10. 1111/pbi.13308.

Xiao SH, Hu Q, Shen JL, et al. GhMYB4 downregulates lignin biosynthesis and enhances cotton resistance to Verticillium dahliae. Plant Cell Rep. 2021a;40:735-51. https://doi.org/10.1007/s00299-021-02672-x.

Xiao SH, Hu Q, Zhang XJ, et al. Orchestration of plant development and defense by indirect crosstalk of salicylic acid and brassinosteorid signaling via transcription factor GhTINY2. J Exp Bot. 2021b;72:4721-43. https:// doi.org/10.1093/jxb/erab186.

Xu L, Zhang WW, He X, et al. Functional characterization of cotton genes responsive to Verticillium dahliae through bioinformatics and reverse genetics strategies. J Exp Bot. 2014;65:6679-92. https://doi.org/10.1093/ jxb/eru393.

$X u$ J, Wang $X Y$, Li YQ, et al. Host-induced gene silencing of a regulator of $G$ protein signalling gene (VdRGS1) confers resistance to Verticillium wilt in cotton. Plant Biotechnol J. 2018;16(9):1629-43. https://doi.org/10.1111/ pbi.12900.

Zhang JF, Yu JW, Pei WF, et al. Genetic analysis of Verticillium wilt resistance in a backcross inbred line population and a meta-analysis of quantitative trait loci for disease resistance in cotton. BMC Genomics. 2015;16:577. https:// doi.org/10.1186/s12864-015-1682-2.
Zhang Y, Liang Z, Zong Y, et al. Efficient and transgene-free genome editing in wheat through transient expression of CRISPR/Cas9 DNA or RNA. Nat Commun. 2016;7:12617. https://doi.org/10.1038/ncomms12617.

Zhang LS, Ni H, Du X, et al. The Verticillium-specific protein VdSCP7 localizes to the plant nucleus and modulates immunity to fungal infections. New Phytol. 2017a;215:368-81. https://doi.org/10.1111/nph.14537.

Zhang T, Zhang BS, Hua CL, et al. VdPKS1 is required for melanin formation and virulence in a cotton wilt pathogen Verticillium dahliae. Sci China Life Sci. 2017b;60:868-79. https://doi.org/10.1007/s11427-017-9075-3.

Zhang JX, Wang FR, Zhang CY, et al. A novel VIGS method by agroinoculation of cotton seeds and application for elucidating functions of GhBl-1 in salt-stress response. Plant Cell Rep. 2018a;37:1091-100. https://doi.org/ 10.1007/s00299-018-2294-5.

Zhang M, Cao YB, Wang ZP, et al. A retrotransposon in an HKT1 family sodium transporter causes variation of leaf $\mathrm{Na}^{+}$exclusion and salt tolerance in maize. New Phytol. 2018b;217:1161-76. https://doi.org/10.1111/nph. 14882.

Zhang ZN, Ge XY, Luo XL, et al. Simultaneous editing of two copies of Gh143-3d confers enhanced transgene-clean plant defense against Verticillium dahliae in allotetraploid upland cotton. Front Plant Sci. 2018c;9:842. https://doi.org/10.3389/fpls.2018.00842.

Zhang WW, Ren YH, Zhang HC, et al. Genetic variations of prevailing Verticillium dahliae isolates from cotton in China. J Plant Pathol. 2019a;101:56578. https://doi.org/10.1007/s42161-018-00236-9.

Zhang YH, Jin YY, Gong Q, et al. Mechanismal analysis of resistance to Verticillium dahliae in upland cotton conferred by overexpression of RPL18A-6 (Ribosomal Protein L18A-6). Ind Crops Prod. 2019b;141:111742. https://doi org/10.1016/j.indcrop.2019.111742.

Zhang JF, Abdelraheem A, Thyssen GN, et al. Evaluation and genome-wide association study of Verticillium wilt resistance in a MAGIC population derived from intermating of eleven Upland cotton (Gossypium hirsutum) parents. Euphytica. 2020;216:9. https://doi.org/10.1007/ s10681-019-2547-6.

Zhang Y, Chen B, Sun ZW, et al. A large-scale genomic association analysis identifies a fragment in Dt1 1 chromosome conferring cotton Verticillium wilt resistance. Plant Biotechnol J. 2021;19:2126-38. https://doi.org/10. 1111/pbi.13650.

Zhao J, Liu JG, XU JW, et al. Quantitative trait locus mapping and candidate gene analysis for Verticillium wilt resistance using Gossypium barbadense chromosomal segment introgressed line. Front Plant Sci. 2018;9:682. https://doi.org/10.3389/fpls.2018.00682.

Zhou X, Stephens M. Genome-wide efficient mixed-model analysis for association studies. Nat Genet. 2012;44:821-4. https://doi.org/10.1038/ng.2310.

Ready to submit your research? Choose BMC and benefit from:

- fast, convenient online submission

- thorough peer review by experienced researchers in your field

- rapid publication on acceptance

- support for research data, including large and complex data types

- gold Open Access which fosters wider collaboration and increased citations

- maximum visibility for your research: over $100 \mathrm{M}$ website views per year

At BMC, research is always in progress.

Learn more biomedcentral.com/submissions 